\title{
Néolithisation et néolithique : Libye et Sahara
}

\author{
Barbara E. Barich
}

\section{OpenEdition}

Journals

Édition électronique

URL : https://journals.openedition.org/encyclopedieberbere/2719

DOI : 10.4000/encyclopedieberbere. 2719

ISSN : 2262-7197

\section{Éditeur}

Peeters Publishers

\section{Édition imprimée}

Date de publication : 5 octobre 2012

Pagination : $5467-5474$

ISBN : 978-90-429-2718-6

ISSN : 1015-7344

\section{Référence électronique}

Barbara E. Barich, « Néolithisation et néolithique : Libye et Sahara », Encyclopédie berbère [En ligne], 34 | 2012, document N42, mis en ligne le 15 décembre 2020, consulté le 17 février 2022. URL : http:// journals.openedition.org/encyclopedieberbere/2719; DOI : https://doi.org/10.4000/ encyclopedieberbere. 2719

Ce document a été généré automatiquement le 17 février 2022.

(c) Tous droits réservés 


\title{
Néolithisation et néolithique : Libye et Sahara
}

\author{
Barbara E. Barich
}

1 Le terme "Néolithique saharien" comprend en général un complexe de traits culturels qui se sont manifestés entre 10000 et 5000 ans dans la partie centrale du Sahara comprise entre $15^{\circ}$ et $25^{\circ}$ Lat. Nord. Parmi les aspects novateurs qui caractérisent ces cultures différents éléments technologiques, aspects artistiques et modèles d'établissements ce sont l'apparition de la céramique et la production de nourriture, avec une économie pastorale qui représentent indubitablement les aspects les plus importants. En ce qui concerne les processus de mise en culture des végétaux, nous n'avons en réalité que quelques preuves d'une utilisation certaine d'espèces appartenant à la famille du millet et du sorgho, et par ailleurs il manque des témoignages attestant de résidences permanentes.

2 Les travaux de la Combined Prehistoric Expedition, conduits par F. Wendorf et R. Schild (2001) ont mis en lumière le rôle moteur des communautés du Désert occidental égyptien, foyer des premières expériences d'une domestication animale, qui se serait diffusée à partir de cette région dans une grande partie des territoires africains, tant à l'ouest vers le Sahara central, qu'à l'est, vers la vallée du Nil. De ce point de vue, et à propos des pratiques naissantes de mise en culture de certains végétaux, d'autres études récentes ont mis en évidence le rôle de ce territoire qui, avec la zone des oasis égyptiennes (Farafra, Dakhla et Kharga) ont du constituer un centre autonome d'expériences proto-néolithiques et de médiation entre le Sahara et la vallée du Nil (Barich 2002).

3 Le thème de la domestication locale du bœuf, à partir de spécimens sauvages de Bos primigenius a été longuement débattu dans la littérature africaniste (Gautier 1987; Smith 2005). La région de Bir Kiseiba en Egypte méridionale a livré plus de 10000 vestiges de faune dont la majorité correspond à des espèces sauvages adaptées au climat aride (lièvre, gazelle). Quelques restes très fragmentaires de bœuf ont été déterminés avec quelques réserves comme spécimen domestique ou tout au moins comme ayant atteint un stade de domestication naissante (Gautier 1984, 2001). Il a 
surtout été observé que les bovidés sauvages (bœufs ou buffles) n'auraient pas pu survivre avec un taux d'humidité aussi faible que celui enregistré aujourd'hui. Actuellement, les nouvelles recherches sur l'ADN mitochondrial du Bos ont révélé une très ancienne séparation (datant d'environ 25000 ans) des spécimens africains par rapport à ceux d'Eurasie et d'Inde (Bradley, Magee 2006), validant l'hypothèse de l'indépendance du processus de domestication bovine en Afrique (Holl 2004). Le phénomène se serait développé pendant la phase initiale de l'Holocène quand, avec le rétablissement du cycle de la mousson et la reprise des pluies d'été, des groupes provenant de la vallée du Nil (probablement issus d'environnement de l'Arkinien) vinrent occuper à nouveau le territoire après des millénaires d'aridité sévère. La constitution de petits troupeaux domestiques aurait favorisé l'exploitation des territoires désertiques en fournissant des ressources de protéines disponibles même au cours des crises climatiques répétées. Cette hypothèse expliquerait également la diffusion plutôt rapide auprès des communautés du Sahara central. En effet, une interruption, relevée dans la séquence saharienne entre ca 6800 et 6500 cal BC (Hassan 2002), est à mettre en relation avec l'intensification parallèle des premières expérimentations de constitution de troupeaux par les groupes du Sahara central occidental.

4 Les données relatives aux communautés holocènes du Sahara central constituent deux catégories de documentation. La plus impressionnante regroupe le vaste répertoire des représentations rupestres sur des thèmes pastoraux, œuvres gravées ou peintes sur les parois des abris sous roche dans le Tassili-n-Ajjer, la Tadrart Acacus, le Messak, et dans l'Ahaggar (Le Quellec 2001 ; Mori 1998 ; Muzzolini 1995 ; Sansoni 1994). La seconde très complexe, rassemble des informations relatives a la culture materielle et aux données archéozoologiques qui proviennent de fouilles régulières sur les principaux sites à la frontière libyco-algérienne: Ti-n-Hanakaten (Aumassip 2001), le complexe du Tin-nTorha, Uan Muhuggiag*, Uan Tabu (Barich 2010a), cette région ayant joué un rôle clé dans le transfert de bétail domestique venant du Sahara oriental vers les zones occidentales.

5 Les séquences chrono-stratigraphiques les plus sures, en Egypte, en Libye et au Niger font remonter les premières occupations du Sahara a la phase initiale de l'Holocène. Pendant la phase de transition entre le Pléistocène final et l'Holocène, on remarque l'affirmation de situations culturelles fortement liées à l'exploitation des ressources aquatiques que l'archéologue Sutton nomma «culture aqualithique» (Sutton 1974). Cette définition fut d'abord critiquée, mais elle apparaît aujourd'hui tout à fait appropriée. Au Mali, une phase lacustre entre ca 7500 et 5800 cal BC a été repérée dans les coupes stratigraphiques des lacs anciens qui bordent les étendues dunaires. Des sédiments finement stratifiés contenant des restes de faune aquatique, coquilles, diatomées (plantes lacustres) sont le signe incontestable de la présence d'anciens lacs permanents. Les vastes cimetières reconnus dans la zone de Taoudenni ont permis d'importantes observations sur les caractéristiques paléoanthropologiques (Dutour 1997).

Dans le Désert occidental égyptien, le Néolithique de type El Nabta (entre 7150 et 6800 cal BC) apparaît comme une re-colonisation de la même région au terme d'une oscillation aride apres le rétablissement d'une nouvelle phase humide. Les meules en pierre deviennent nombreuses, et des restes de graines de céréales sauvages (sorgho, millet) ont été recueillis lors de fouilles récentes. Durant cette période, des pluies plus 
régulières ont favorisé un type d'établissement plus stable et meme l'apparition d'un vrai village permanent. Nabta E-75-6 présente deux alignements de structures - traces de cabanes, foyers, puits de stockage - selon un schéma qui rappelle celui de Ti-n-Torha Est dans l'Acacus, en Libye. De nombreuses comparaisons ont été établies entre l'industrie lithique trouvée sur le site E-75-6 et celles d'autres sites du Désert occidental, tels que Wadi Bakht, dans le Gilf Kebir, Abu Ballas et Lobo en face du Great Sand Sea (Gehlen et al. 2002), le niveau Masara B à Dakhla (McDonald 2009), et le niveau Holocène Ancien à Farafra (Barich sous presse). Les restes d'ossements de bovin domestique sont rares sur le site E-75-6, de plus, les caprinae apparaissent très tardivement dans la séquence de Nabta. Ils ont été enregistrés sur le site E-75-8 dans des niveaux datés à < $6000 \mathrm{BC}$, et figure parmi les plus anciennes connues en Afrique.

7 Les voies de migration suivies par cette espèce pour atteindre les régions côtières de l'Afrique du Nord restent encore inconnues. Pour la Cyrénaïque, la présence d'animaux domestiques dans l'horizon néolithique de Haua Fteah (McBurney 1967), et les découvertes récentes faites à Abou Tamsa (De Faucamberge 2010), semblent résulter d'une introduction de ces animaux depuis les territoires du Proche-Orient, par le Delta du Nil. En effet, la nature différente des événements néolithiques septentrionaux et les voies de propagation du bétail domestique constituent avec les données chronologiques disponibles (sur patelle et non charbons) un ensemble distinct d'informations sans rapport, semble-t-il pour le moment, avec les communautés du Sahara. Ce sujet s'inscrit dans une autre problématique ouvrant une nouvelle discussion.

8 En Libye tassilienne, la séquence d'occupation principale reconnue dans le massif de la Tadrart Acacus constitue, avec le massif algérien du Tassilin-Ajjer, le complexe d'art rupestre le plus important $\mathrm{du}$ Sahara. Une importante séquence chronostratigraphique a été établie dans l'Oued Tin-Torha, à partir de trois sites, qui, dans leur ensemble, illustrent le peuplement local durant les phases anciennes de l'Holocène. Tin-Torha Est se présente comme un véritable proto-village avec une série de fondations de cabanes adossées à la paroi rocheuse de l'Oued (Barich 1998). Le spectre faunique qui comprend des espèces sauvages (mouflons, gazelles, lièvres), des restes de poissons (Clarias sp. et Tilapia) ainsi que des oiseaux, atteste de la présence d'une économie de chasse-pêche-cueillette. Il convient d'autre part de souligner la présence de restes mal conservés d'un bovin de grande taille (niveaux supérieurs de Tin-n-Torha Est que l'on date entre 7550 et $6000 \mathrm{cal}$ BC), pour lequel A. Gautier, proposa de façon non certaine, l'identification comme bœuf domestique (Gautier, Van Neer 1977-82). La présence de Bos depuis des niveaux aussi anciens dans la séquence Acacus n'est cependant pas confirmée par les recherches successives. La série stratigraphique que l'on obtient à partir des trois sites de l'Oued Tin-Torha (abri Est, abri des Deux Grottes et abri Nord) embrasse tout l'intervalle depuis le début jusqu'à la moitié de l'Holocène.

Dans l'abri de Ti-n-Torha Nord, les deux niveaux supérieurs de la séquence (I-II : 4911-4793 cal BC, R-1031 alfa ; 4273-4146 cal BC, R-1030) montrent la présence de bœuf domestique (Bos taurus) au sein d'un établissement d'occupation qui utilise l'abri pour de brèves périodes de transhumance, liées aux exigeances des troupeaux. La céramique ainsi que les outils lithiques qui ont été recueillis montrent la continuité de certaines techniques de base, bien que ces deux catégories de documents démontrent un appauvrissement du style. Selon la reconstruction proposée par l'auteur (Barich 1987), les trois sites de Ti-n-Torha permettent de reconstruire une séquence de développement local montrant un schéma d'organisation de type encore 
«Paléolithique tardif», tendant vers une organisation économique et sociale plus complexe.

Partant de ce schéma chronologique, et comportemental, on remarque un intervalle chronologique important entre les premiers restes de bœuf «supposé » domestique du Désert occidental et ceux signalés d'autres régions sahariennes et nord-africaines. Au Niger, par exemple, les sites de Dogomboulo dans le secteur de Fachi (ca 6000-5500 cal $\mathrm{BC}$ ) et de Rocher Toubeau ( $c a$ 4500-4300 cal BC), sont les premiers exemples d'une occupation fondée sur l'élevage durant l'Holocène moyen. Le complexe lithique de cette période (le Ténéréen) se caractérise par de grands outils bifaces tels que les gouges (hachettes sur éclats), des disques, des pointes de flèches et des hachettes polies. Un squelette pratiquement complet de bœuf domestique (Bos taurus), retrouvé à Agoras-nTast a été daté entre 5300 et $3900 \mathrm{cal} \mathrm{BC}$. On trouve dans la même région des traces d'activités de récoltes de végétaux sauvages fournies par les empreintes de Brachiaria et de sorgho conservées dans l'argile des vases.

11 Au Niger les recherches de J. P. Roset au cours des années 1980 ont posé les bases stratigraphiques permettant une reconstruction régionale adéquate; ici l'horizon pastoral ne semble pas avoir une origine locale (Roset 1987, 2000). Les sites plus ou moins contemporains de Temet 1 (datés entre 9200 et $9000 \mathrm{cal} \mathrm{BC}$ ); Ti-n-Ouaffadene ( $c a$ 8650-8250 cal BC) et Adrar Bous 10 (8400-7700 cal BC), sont tous liés à l'expansion des lacs durant une phase de précipitations intenses. Le complexe lithique de ces contextes est bien illustré par Temet 1 , le site le plus ancien. Il s'agit d'un complexe épipaléolithique, toutefois, l'outillage épipaléolithique est ici associé à une tradition céramique qui malgré son ancienneté, présente des répertoires décoratifs d'une remarquable richesse. Dans les montagnes Bagzane, le site de Tagalagal a fourni une autre série de dates anciennes pour un niveau avec céramique : de 8800 jusqu'à $8300 \mathrm{cal}$ $B C$. La céramique de Tagalagal présente des variations de forme et une décoration bien développée. On remarque en particulier le motif des «vagues pointillées » (dotted wavy line) largement diffusé parmi les communautés sahariennes de l'Holocène. Les restes fauniques concernent tous des animaux sauvages et des poissons, alors que l'abondance des meules atteste de l'importance de la cueillette des espèces végétales.

La situation observée en Libye, dans l'abri de Uan Muhuggiag* (Barich 2010b), offre quelques arguments pouvant clarifier ces problématiques. Le modèle économique des niveaux inférieurs de Uan Muhuggiag indique une économie pastorale, encore associée à la chasse et à la cueillette de plantes sauvages. C'est cet aspect particulier qui rend le site si important pour la compréhension de la transition vers une économie pleinement pastorale. En effet, bien que les restes de faune de Uan Muhuggiag comprennent encore des espèces sauvages (lièvres, dassies ou rock hyrax, chats sauvages, phacochères, gazelles, mouflons), leur fréquence est très faible $(4,2 \%)$ par rapport aux restes domestiques (92,4\%). La présence de bœuf domestique est attestée depuis le VIII ${ }^{\mathrm{e}}$ millénaire non cal. BP. La technologie comme les données environnementales de Uan Muhuggiag fournissent des témoignages clairs d'un développement progressif et indépendant $\mathrm{du}$ pastoralisme qui prend son origine très certainement au cœur du système saharien. Ce changement de style de vie ne peut toutefois pas être interprété, dans ce cas, comme l'effet d'une intrusion extérieure, mais s'explique plutôt comme la conséquence d'une meilleure interaction entre les groupes humains et leur environnement. Des précisions ultérieures ont été apportées à propos des développements plus tardifs du pastoralisme saharien avec les recherches récentes 
dans l'Acacus (Cremaschi, Di Lernia 1998; Di Lernia 2002) qui montrent une vaste diffusion des sites depuis l'Erg Uan Kasa jusqu'au Tanezrouft, après 6000 non cal. BP. De nombreux sites de plein air, véritables villages, ont été reconnus dans l'Erg Uan Kasa ainsi que dans l'Edeyen Murzuq, le long des rives d'anciens lacs qui avaient alors atteint leur expansion maximum. Les restes de bovins domestiques sont très nombreux dans ces sites, ce qui tend à prouver que ces villages pouvaient être des zones d'occupation principale, habitées toute l'année et point de départ de déplacements saisonniers pour une partie du groupe accompagnée de bétail de petite taille jusqu'aux zones de pâturages alors disponibles dans le massif. La culture pastorale tardive de l'Acacus (Late Pastoral) peut être datée entre ca 3800 et 2200 cal. BC. Après cette date, le territoire semble être abandonné, à l'exception de campements éphémères et transitoires. Le modèle économique est alors principalement fondé sur l'élevage caprin-ovin et sur l'usage des produits secondaires. La céramique de cette période est principalement non décorée, de couleur brune ou rougeâtre, ou bien ornée des motifs imprimés près de l'ouverture du récipient. Parmi les objets manufacturés, les haches de pierre volcanique - jusque là «exotiques" - et les objets bifaces pré-dynastiques attestent de la circulation des groupes sur des distances atteignant des centaines de kilomètres. Pendant la période comprise entre 4900 et $3700 \mathrm{cal} \mathrm{BC}$, la culture pastorale se diffuse dans le Sahara central. Partout, la présence de bergers est associée à d'admirables œuvres d'art rupestre qui semblent avoir été au moins en partie les marqueurs de lieux sacrés où se tenaient des cérémonies d'initiation.

On ne connaît pas en revanche de témoignages de la présence de bétail domestique dans les marges nord-occidentales du Sahara, dans la région située entre les zones algériennes et marocaines au sud de l'Atlas et de l'Aurès, et le Tanezrouft, c'est-à-dire à la limite entre le Mali et l'Algérie. Cette région semble avoir été habitée par des groupes mobiles de chasseurs-cueilleurs peut-être porteurs encore de traditions capsiennes (cultures de Hassi el-Abiod, culture de Kobadi) (Raimbault, Commelin 2001-2002). Sur la base de ces preuves, il semble possible d'affirmer que la portion nord-occidentale du Sahara ne fit pas office de couloir de passage pour diffuser le mouvement pastoral dans les régions atlasiques du nord (Aurès) et de l'ouest. Au contraire, les premiers sites sahariens ayant une faune domestique qui datent de la période 4900-3700 cal BC (comme le célèbre Adrar Bous au Niger) sont distribués sur le versant occidental du Ténéré. Ce serait probablement à partir de là que se diffusèrent les faunes domestiques vers le sud et le sud-ouest atteignant la côte atlantique du Sahara occidental, à Tintan et Chami, le bassin du Taoudenni à Erg In-Sakane, l'Adrar des Ifoghas et la vallée du Tilemsi au Mali ; Dhar Tichitt en Mauritanie ; la vallée de l'Azawagh au Niger jusqu'à Kintampo et Ntereso dans la région du Ghana.

\section{BIBLIOGRAPHIE}

AUMASSIP G., 2001 - L'Algérie des premiers hommes, Paris, Ed. Maison des Sciences de l'Homme. 
BARICH B. E. (ed.), 1987 - Archaeology and environment in the Libyan Sahara: The excavations in the Tadrart Acacus, 1978-1983, Oxford, British Archaeological Reports, International Series : 368.

BARICH B. E., 1998 - People, water and grain - The beginnings of domestication in the Sahara and the Nile Valley, Roma, L’Erma di Bretschneider.

BARICH B. E., 2002 - "Cultural responses to Climatic Changes in North Africa", in Droughts, Food and Culture - Ecological Change and Food Security in Africa's Later Prehistory, Hassan F.A. (ed.), New York, Kluwer Academic/Plenum Publishers, p. 209-223.

BARICH B. E., 2010a - Antica Africa - Alle origini delle società, Roma, L'Erma di Bretschneider.

BARICH B. E., 2010b - “Uan Muhuggiag”. in Encyclopédie Berbère, fasc. XXXII, Paris-Louvain-Walpole, Peeters, p. 5101-5104.

BARICH B. E. sous presse - "Early to Mid-Holocene occupation of the Egyptian Western Deserf, in From Lake to sand - The archaeology of Farafra oasis, Barich B.E., Lucarini G., Hassan F.A., Hamdan M.A.(eds.), Firenze, All'Insegna del Giglio.

BRADLEY D. G., MAGEE D., 2006 - "Genetics and the origins of domestic cattle", in Zeder M.A., Emshwiller E., Smith B.D., Bradley D.G. (eds.), Documenting Domestication : new genetics and archeological paradigm, California, University of California Press, p. 317-328.

CREMASCHI, M., DI LERNIA, S., 1998 - Wadi Teshuinat, Palaeoenvironment and Prehistory in South-western Fezzan, Firenze, Edizioni all'Insegna del Giglio.

DI LERNIA S., 2002 - "Dry climatic events and cultural trajectories : adjusting Middle Holocene pastoral economy of the Libyan Sahara", in Droughts, Food and Culture - Ecological Change and Food Security in Africa's Later Prehistory, Hassan F.A. (ed.), New York, Kluwer Academic/Plenum Publishers, p. 225-250.

DUTOUR O., 1997 - «Peuplement du Sahara au Pleistocène Supérieur, le point de vue Paléoanthropologique », in Sahara. Paléomilieux et Peuplement Préhistorique au Pléistocène Supérieur, Tillet T. (ed.), Paris, L'Harmattan, p. 409-421.

DE FAUCAMBERGE E., 2010 - Abou Tamsa : étude d'un nouveau site néolithique en Cyrénaïque (Libye), Thèse de Doctorat, Université de Paris I (Panthéon-Sorbonne), 351 p.

GAUTIER A., 1984 - “Archaeozoology of the Bir Kiseiba region, Eastern Sahara”, in Cattle-Keepers of the Eastern Sahara, Wendorf F., Schild R. (assemblers), Close A.E. (ed.), Dallas, Department of Anthropology, Institute for the Study of Earth and Man, Southern Methodist University, p. 49-72. GAUTIER A., 2001 - "The Early to Late Neolithic archeofaunas from Nabta and Bir Kiseiba”, in Holocene Settlement of the Egyptian Sahara - Vol. I : The Archaeology of Nabta Playa, Wendorf F., Schild R. and Associates, New York, Boston, Dordrecht, London, Moscow, Kluwer Academic/Plenum Publishers, p. 609-635.

GAUTIER A., VAN NEER W., 1977-82 - "Prehistoric Fauna from Ti-n-Torha (Tadrart Acacus, Libya)", Origini XI, p. 87-127.

GEHLEN B., KINDERMANN K., LINSTADTER J., RIEMER H., 2002 - “The Holocene occupation of the Eastern Sahara", in Tides of the Desert, Jennerstrasse 8 (ed.), Africa Praehistorica 14, Köln, Heinrich Barth Institut, p. 85-116,.

HASSAN F. A., ed. 2002 - Droughts, Food and Culture - Ecological change and food security in Africa's later prehistory. New York, Boston, Dordrecht, London, Moscow, Kluwer Academic/Plenum Publishers. 
HOLL A., F.C., 2004 - Holocene Saharans - An Anthropological Perspective, London-New York, Continuum.

LE QUELLEC J.-L. (ed.) 2001 - Ithyphalliques, traditions orales, monuments lithiques et art rupestre au Sahara. Saint-Lizier, AARS.

MCBURNEY C.B.M., 1967 - The Haua Fteah (Cyrenaica) and the Stone Age of the South-East Mediterranean, Cambridge, Cambridge University Press.

MC DONALD M.-M.A., 2009 - "Increased sedentism in the central oases of the Egyptian Western Desert in the early to mid-Holocene : Evidence from the peripheries", African Archaeological Review 26, p. 3-43.

MORI F., 1998 - The Great Civilisations of the Ancient Sahara. Roma, L'Erma di Bretschneider.

MUZZoLINI A., 1995 - Les images rupestres du Sahara. Toulouse, Collection Préhistoire du Sahara.

RAIMBAult M., COMmelin D., 2001-2002 - « La poterie du site néolithique de Kobadi dans le Sahara malien », Préhistoire Anthropologie Méditerranéenne, t. 10-11, p. 107-116.

ROSET J.P., 1987 - "Palaeoclimatic and cultural conditions of Neolithic development in the early Holocene of Northern Niger (Aïr and Ténéré)", in Prehistory of Arid North Africa, Close A.E. (ed.), Dallas, Southern Methodist University Press, p. 211-234.

ROSET J.P., 2000 - « Céramique et néolithisation en Afrique Saharienne, in Premiers paysans du monde - Naissances des agricultures, sous la dir. J. Guilaine, Paris, Editions Errance, p. 263-290.

SANSONI U., 1994 - Le più antiche pitture del Sahara. L'arte delle Teste Rotonde, Milano, Jaca Book.

SMITH A.-B., 2005 - African Herders : Emergence of Pastoral Tradition, Walnut Creek, AltaMira Press.

SUTTON J.-E.G., 1974 - “The aquatic civilization of middle Africa”, Journ. of African History 15, p. 527-546.

WENDORF F., SCHILD R. and Associates, 2001 - Holocene Settlement of the Egyptian Sahara - Vol. I : The Archaeology of Nabta Playa, New York, Boston, Dordrecht, London, Moscow, Kluwer Academic/ Plenum Publishers.

\section{INDEX}

Mots-clés : Art rupestre, Libye, Néolithique, Sahara 